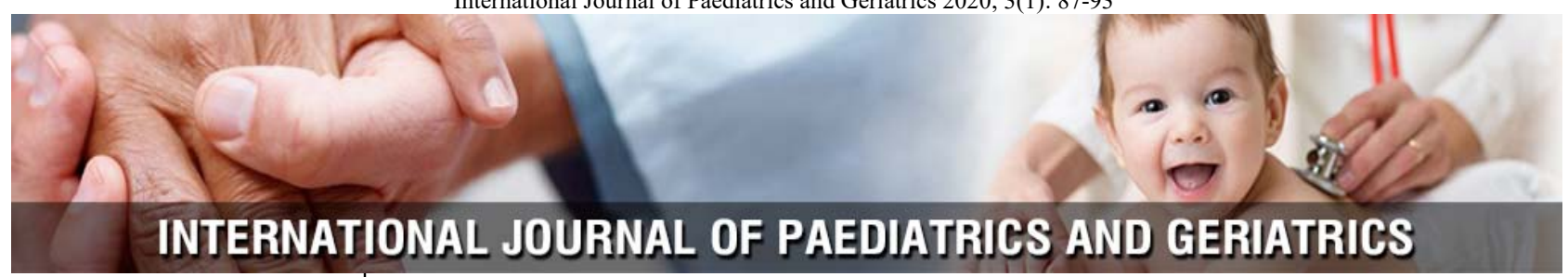

P-ISSN: 2664-3685 E-ISSN: $2664-3693$

www.paediatricjournal.com IJPG 2020; 3(1): 87-93

Received: 14-11-2019

Accepted: 18-12-2019

Muralidhar Reddy K Consultant Pediatrician, Sangamitra Hospital, Ongole, Andhra Pradesh, India

Tatam Naidu N

Consultant Pediatrician, Sangamitra Hospital, Ongole, Andhra Pradesh, India
Corresponding Author: Muralidhar Reddy K Consultant Pediatrician, Sangamitra Hospital, Ongole, Andhra Pradesh, India

\section{Optimal nutrition in gastrointestinal distress among infants aged 6-8 weeks: An observational study in India}

\author{
Muralidhar Reddy K and Tatam Naidu N
}

DOI: https://doi.org/10.33545/26643685.2020.v3.i1b.65

\begin{abstract}
The ambiguity among the gastrointestinal (GI) disease symptoms and aetiology in apparently healthy, well-fed term infants make the diagnosis and interventions of GI disease challenging. We assessed if there was a difference in the GI tolerance of lactose predominant formula-fed and human-milk fed infants. The study divided mother and infant (6-8 weeks of age) pairs into 'predominantly breastfeeding (BF)' and 'predominantly infant formula (IF)' feeding groups. The Infant Gastrointestinal Symptom Questionnaire (IGSQ-13) index score and symptom-wise (stooling, spitting/vomiting, crying, fussiness, and flatulence) analysis showed no statistically significant difference between the study groups. The role of optimal nutrition which is closer to breastmilk in providing gut comfort has been elicited by this study. $100 \%$ lactose-based formula can be considered as a promising alternative to the management of infants with GI distress, however, IF should always be considered as a substitute and not a replacement to breastmilk.
\end{abstract}

Keywords: Breast milk, gastrointestinal (GI) distress, nutrition, infant formula, regurgitation

\section{Introduction}

Functional gastrointestinal (GI) symptoms experienced during infancy can be attributed to the physiological development or from the maladaptive behavioral responses to the internal or external stimuli ${ }^{[1]}$. Common causes of GI distress noted during infancy include colic, GI reflux (GER), and functional constipation. Although GI distress is minor and self-limiting, it often results in hospitalization, feeding changes, medications and emotional distress to parents ${ }^{[2]}$.

It is estimated that the global prevalence of infantile colic is $\sim 10 \%-40 \%$ with a peak prevalence at 6 weeks ${ }^{[3]}$. Constipation manifests in $\sim 17 \%-40 \%$ infants during the first year of life, whereas, $70 \%-85 \%$ infants have regurgitation within the first two months of life ${ }^{[4,5]}$. A review by Poddar et al. indicated that nearly $50 \%$ of healthy Indian infants regurgitate at least once a day for the first 4 months, which gradually declines in $90 \%$ of infants by 1 year of age ${ }^{[6]}$. Although the global prevalence of GI distress among infants is higher, the etiologies of colic, GER, and constipation remain largely unknown. Studies have proposed various causes including intolerance to cow's milk protein or lactose; GI immaturity or inflammation; increased serotonin secretion; poor feeding technique; and maternal smoking or nicotine replacement therapy ${ }^{[3]}$.

Gastrointestinal distress comprises a range of disorders that are related to the GI tract; however, there are no specific biochemical markers or structural abnormalities, which can help in the diagnosis and progression of these disorders ${ }^{[7]}$. In addition, through the first years, children cannot express their symptoms and hence the decision to seek medical attention is dependent on caregiver's previous experiences, expectations, and perception of illness, which the makes diagnosis of such diseases further challenging ${ }^{[7,8]}$. For the past decade, the diagnosis of GI disorders in children of all ages was based on the symptom-based

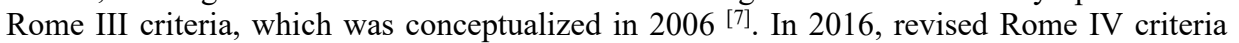
were proposed, which clearly distinguished the GI disorders in young (neonates/toddlers) and older children. Rome IV criteria has made notable revisions in the diagnostic criteria for infant colic and other gastrointestinal disorders to facilitate the diagnosis and timely management in children [7]. However, only recently, a joint recommendation of the North American Society for Pediatric Gastroenterology, Hepatology, and Nutrition and the 
European Society for Pediatric Gastroenterology, Hepatology, and Nutrition (ESPGHAN) have published their guidelines on managing pediatric gastroesophageal reflux in clinical practice ${ }^{[9]}$.

The current treatment management in infants with GI distress includes continued $\mathrm{BF}$, change in formula, complementary feeding, and so on ${ }^{[3,8]}$. However, because of varied etiology, symptomatic representation, and lack of guidelines on definitive management, healthcare providers are still in search of the appropriate strategies that can help diagnose and manage infantile GI distress effectively ${ }^{[10]}$. Conversely, inappropriate diagnosis and management can cause unnecessary physical and emotional distress [8]. Emerging evidence has shown that effective nutritional strategies can be a promising option for prevention and management of GI symptoms ${ }^{[10,11]}$.

In this regard, the current study was proposed to evaluate the role of optimum nutrition in gut comfort, with the hypothesis that there is no difference in GI tolerance of lactose- predominant formula-fed and human-milk fed infants as measured with the GI index score.

\section{Aim and Objective}

The primary objective of this study was to evaluate the effect of different feeding practices (predominant infant formula (IF) feeding, predominantly breast milk (BM) feeding, and mixed feeding BM and IF/animal milk) on GI tolerance and symptom burden measured using the Infant Gastrointestinal Symptom Questionnaire (IGSQ-13) in infants aged 6-8 weeks.

The secondary objective was to characterize the effect of feeding mode (e.g., predominant IF feeding, predominantly $\mathrm{BM}$ feeding, and mixed feeding $\mathrm{BM}+\mathrm{IF} /$ animal milk) in infants at 6-8 weeks of age.

\section{Materials and Methods}

This observational study was conducted over a period of 4 months in the Pediatric outpatient clinic of India. The study was approved by the Institutional ethical committte.

\section{Participant enrollment and selection}

Mother and full-term infants aged 6-8 weeks, who have been visiting the pediatrician's outpatient department, were enrolled in the study. Writen informed consent was acquired from all the enrolled participants before beginning the study. The following information was obtained from the enrolled participants:

a. General infant characteristics, including anthropometric measurements (age, weight, and length)

b. Prior history of episodes of illness and gut comfort (absence of colic, regurgitation, and constipation was assessed with Rome IV criteria)

c. Sociodemographic information of mother and child pair

d. Feeding history/pattern indicating the intake of breast milk, IF, animal milk, and others.

Only full-term (37-42 weeks of gestation) infants who either have been predominantly receiving $\mathrm{BF}$, formula feeding (IF), or mixed feeding (including animal milk; $\mathrm{BM}+\mathrm{IF}$ ) were included in the study. Participants suffering from chronic illness or acute recent illness (in the last 2 weeks) requiring medical follow-up or with a known history of milk allergy were excluded from the study. Caregivers or mothers who were not likely to comply with the instructions or study procedure or those who had participated in another clinical trial during the last 4 weeks prior to the beginning of this study were also not included in the study.

\section{Study groups}

For this study, the 'predominenetly BF group' was defined as no more than two feedings of IF or other milks in a day. The "predominently IF group" was defined as no more than two feedings of BM or other milks per day. A schematic flow of the study is indicated in Figure 1. We also enquired about the various IF brands mothers were using for feeding their infants.

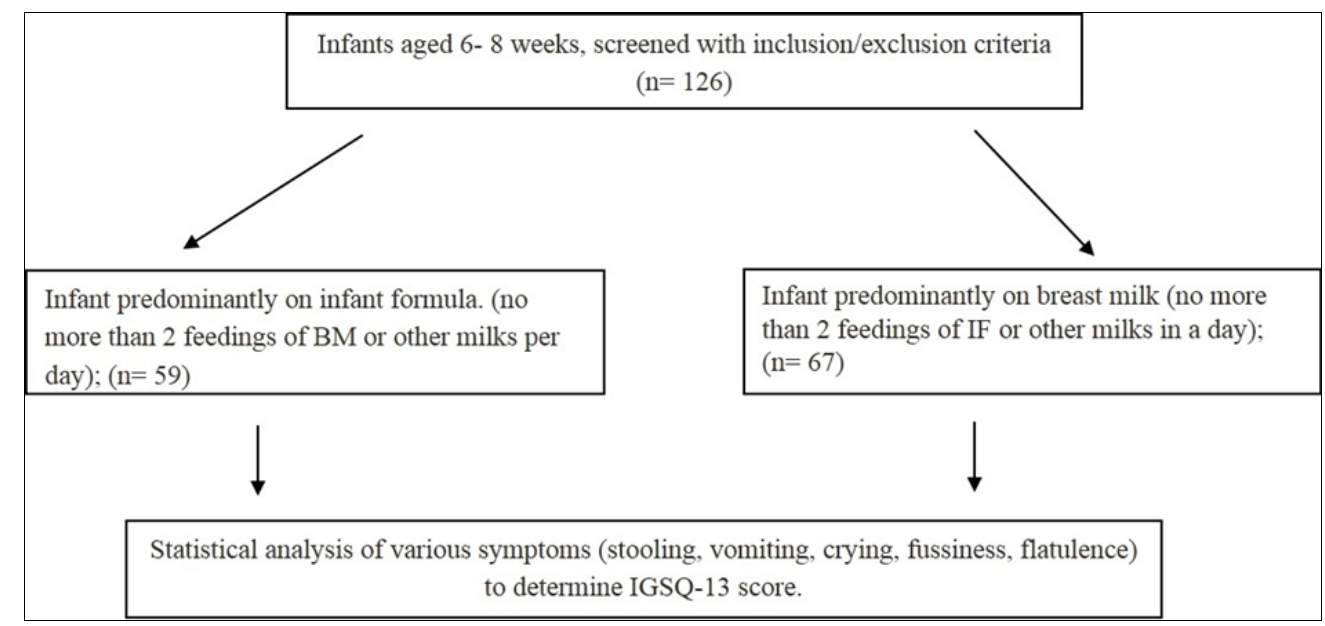

Fig 1: An overview of the study schematic

\section{Determining the IGSQ Score}

All the participants selected for the study were asked to fill up a questionnaire/survey at the end of the study, i.e., approximately at $6-8$ weeks from the starting of the study to determine the symptom burden measured using the IGSQ13 index score.
The IGSQ was developed based on the previously published study by Riley et al. to assess the feeding tolerance in infants based on the parents' perception ${ }^{[12]}$ We developed a questionnaire that consisted thirteen questions testing the following five symptom domains: stooling (two questions), spitting-up/vomiting (four questions), crying (three 
questions), fussiness (two questions), and flatulence (two questions). Please refer to the supplementary section for the set of questions used for recording discrete and numeric responses of the participants.

The participants were asked to fill up two types of questions in the IGSQ questionnaire. In the first set of questions, the mothers had to choose a discrete option out of the six options provided for that question. The other set of questions asked the mothers to fill in a numeric response. All the numeric responses were recorded between 1 and 5 for summarization and analysis. The first five categories of the responses indicated 1 as the absence of symptoms, while 5 indicated high frequency, intensity, or duration of the symptoms. In the end, the Index IGSQ score was obtained by adding the scores of all 13 individual items. The IGSQ was determined for both IF and BF study groups.

\section{Statistical analysis}

The mother's discrete responses to the 13 questions were summed to calculate the percentage with respect to the total responses received for that study group. Continuous data were presented by descriptive statistics i.e. N, mean, standard deviation, minimum and maximum values. Categorical data were presented as frequency count and percentage. Analysis of variance (ANOVA) was used to compare the mean scores between $\mathrm{BF}$ and IF groups. Two sample t-test was used to perform comparison between $\mathrm{BF}$ and IF in different brands. All statistical tests were performed using significance level of $5 \%$.

\section{Results}

\section{Participants' characteristics}

The total number of eligible participants for this study were 126 mother -infant pairs, among whom 59 (46.8\%) infants predominantly received IF and $67(53.2 \%)$ infants predominantly received $\mathrm{BF}$, as indicated in Figure $2 \mathrm{~A}$. The mean age of all the infants was $6.69 \pm 1.54$ weeks and the mean weight was $2.83 \pm 0.55 \mathrm{~kg}$. The anthropometric measurements of all the infants were comparable to to the mean weight of $4.14 \pm 0.55 \mathrm{~kg}$ and height of $73.74 \pm 7.11 \mathrm{~cm}$ at the beginning of the study as shown in Figure 2B. Furthermore, no gender differences were noted with respect to feeding practices, i.e., either IF or BF (Figure 2C).
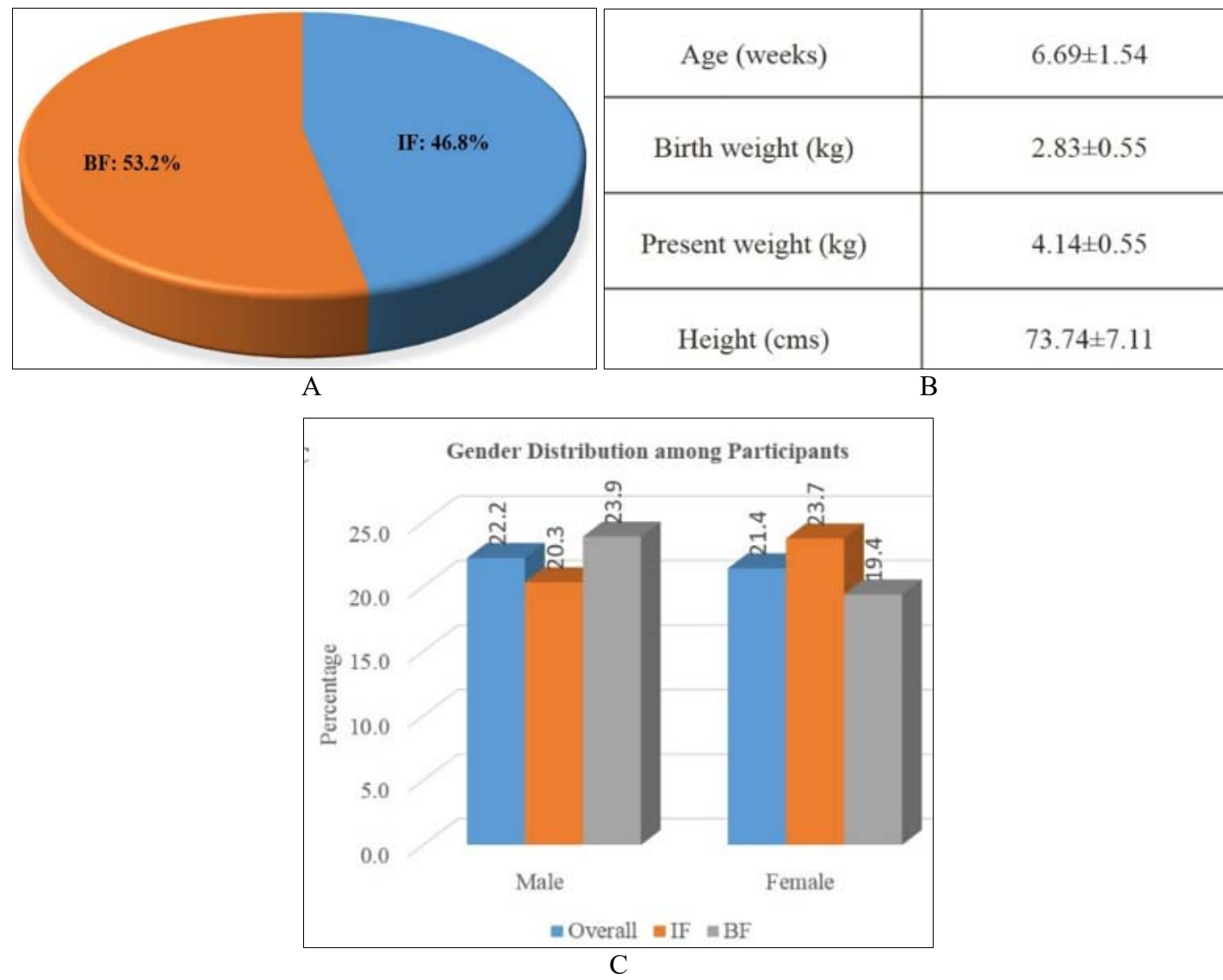

Fig 2: Participants' characteristics ( $n=126)$. (A) Percentage of the participants predominantly receiving either infant formula (IF) or breastfeeding (BF). (B) The anthropometric measurements of all the infants. (C) Gender distribution among the study participants

\section{Mothers' or Guardians’ Level of Education}

The participants' (mother-infant pair) demographic characteristics were recorded for both IF and BF study groups. Participants' mothers' highest education and employment status were recorded as indicated in Table 1. It was observed that $71.4 \%$ of mothers had finished their graduation and $81.7 \%$ of the participants were full-time homemaker. About $4 \%-18 \%$ of the mothers indicated that they were either full- or part-time employee, as indicated in Table 1. 
Table 1: Mothers'/Guardians' Highest Level of Education

\begin{tabular}{|c|c|c|c|c|c|c|}
\hline What is mother / guardian's highest level of education? & Overall N\% & $\mathbf{N}$ & $\mathbf{I F} \%$ & $\mathbf{N}$ & BF ok, \\
\hline Secondary stage & 5 & 4.0 & 9 & 15.3 & 7 & 10.4 \\
\hline Sr. Secondary stage & 16 & 12.7 & 0 & 0.0 & 5 & 7.5 \\
\hline Graduation & 90 & 71.4 & 44 & 74.6 & 46 & 68.7 \\
\hline Post-graduation & 8 & 6.3 & 3 & .5 .1 & 5 & 7.5 \\
\hline Uneducated Employment status & 3 & 2.4 & 1 & 1.7 & 2 & 3.0 \\
\hline Employed/Self-employed Full-Time & 18 & 14.3 & 9 & 15.3 & 9 & 13.4 \\
\hline Employed/Self-employed Part-Time & 4 & 3.2 & I & 1.7 & 3 & 4.5 \\
\hline Unemployed, looking for work & 0 & 0.0 & 0 & 0.0 & 0 & 0.0 \\
\hline Homemaker & 103 & 81.7 & 49 & 83.1 & 54 & 80.6 \\
\hline Student & 0 & 0.0 & 0 & 0.0 & 0 & 0.0 \\
\hline Disabled/Unable to work & 0 & 0.0 & 0 & 0.0 & 0 & 0.0 \\
\hline
\end{tabular}

Infants' characteristics with respect to their health status and medical history (immunization schedule, fever, bowel movement, and so on) were recorded as indicated in Table 2. Overall, $98.4 \%$ infants were healthy, whereas only $68.3 \%$ of the infants had received their immunization on time (as per their age). All the health-related characteristics were comparable between the two study groups.

\section{Feeding Practices}

The mothers were also asked about the feeding pattern, as indicated in Table 3. Around 98\% mothers from the BF group breastfed their infants, whereas $100 \%$ of mothers from the IF group fed their infants with one of the branded IFs available in the market. None of the mothers indicated feeding their infants with animal milk or a combination of IF and animal milk.

Table 3: Feeding Status of the participant

\begin{tabular}{|c|c|c|c|c|c|c|}
\hline & \multicolumn{2}{|c|}{ Overall } & \multicolumn{2}{|c|}{ IF } & \multicolumn{2}{|c|}{ BF } \\
\hline & $\mathbf{N}$ & $\%$ & $\mathbf{N}$ & $\%$ & $\mathbf{N}$ & $\%$ \\
\hline \multicolumn{7}{|c|}{ Have you breast feed you infant in the last week? } \\
\hline Yes & 101 & 80.2 & 35 & 59.3 & 66 & 98.5 \\
\hline No & 1 & 0.8 & 1 & 1.7 & 0 & 0.0 \\
\hline \multicolumn{7}{|c|}{ In the past week, how many times in a typical day $(24 \mathrm{~h})$ did you feed your child? } \\
\hline Infant Formula & 67 & 53.2 & 59 & 100.0 & 8 & 11.9 \\
\hline Animal milk & 0 & 0.0 & & 0.0 & & 0.0 \\
\hline Infant formula \& Animal milk & 0 & 0.0 & & 0.0 & & 0.0 \\
\hline
\end{tabular}

It was observed that overall $46 \%$ of mothers preferred $100 \%$ lactose based formula and $4 \%$ mothers fed their infants with Nan IF, as indicated in Figure 3. Around $88.1 \%$ of mothers from the IF group indicated using lactose based IF over other brands. Even $9 \%$ of mothers from the BF group used lactose based formula.

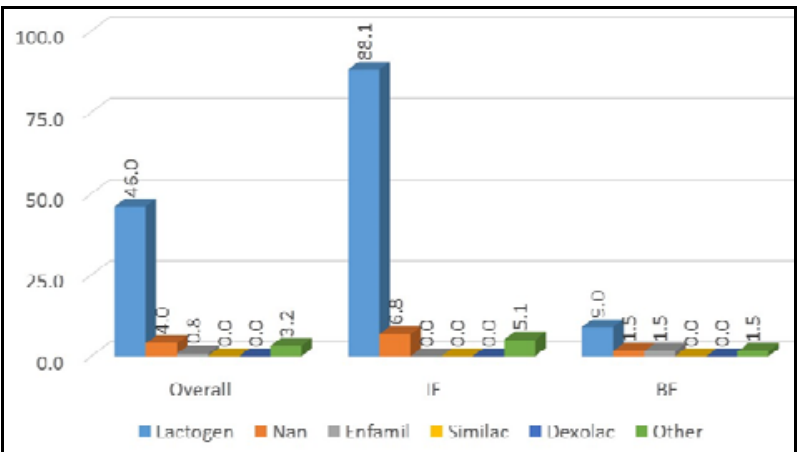

Fig 3: Maximum number of mothers from both the groups chose $100 \%$ lactose based formula for feeding their infants.

\section{The index IGSQ score and GI disease burden}

The numeric responses filled up by the mothers to indicate the GI burden were added together in case of BF or IF study groups to determine the IGSQ-13 score. The cumulative scores of all the 13 questions for the IF and BF groups were similar and not statistically significant. In addition, symptom-wise (stooling, spitting/vomiting, crying, fussiness, and flatulence) analysis showed no significant difference between the groups, as indicated in Figure 4.

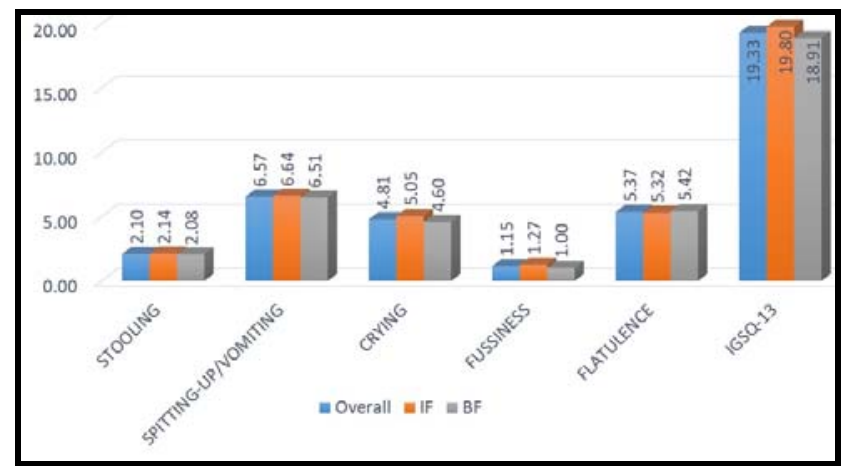

Fig 4: Overall IGSQ score and symptom-based IGSQ score for both, IF and BF, study groups

Furthermore, the IGSQ-13 score showed no statistically significant difference when the $100 \%$ lactose based group was compared with $\mathrm{BF}$ group, as indicated in Figure 5A. We did not find any statistically significant difference in the symptom-wise score and the IGSQ-13 score for Nan IFreceived infants when compared with the BF group (Figure 5B). Similarly, no statistically significant IGSQ-13 score and symptom wise scores were observed for infants receiving combined IF (lactose based and/or Nan) and breastfed infants (Figure 5C). 


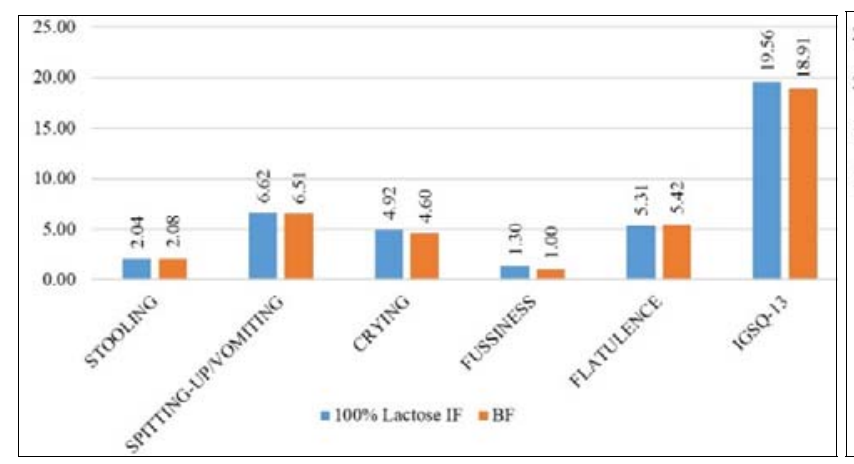

A

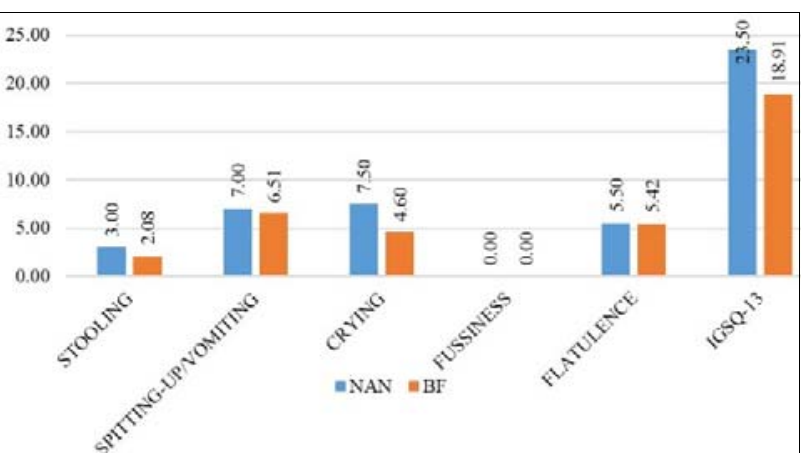

B

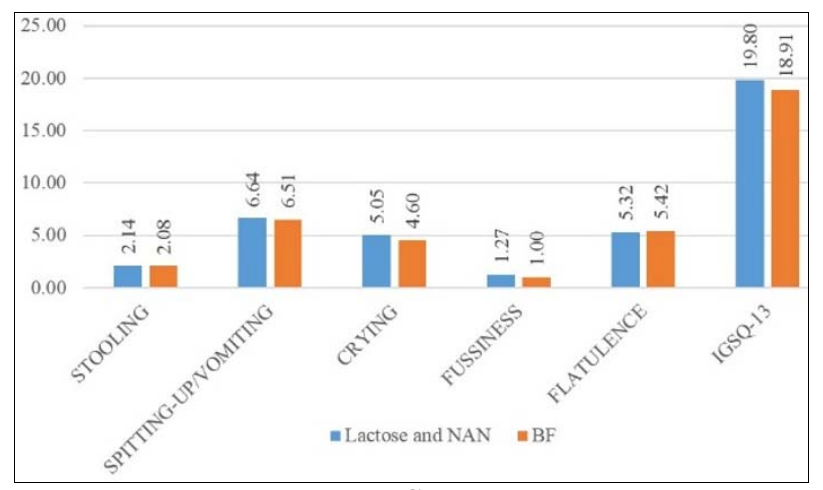

C

Fig 5: Comparison IGSQ-13 and symptom-wise scores for various experimental conditions. (A) IGSQ-13 score and symptom-specific score for lactose based group vs. BF group infants. (B) IGSQ-13

Score and symptom-specific score for the Nan group vs. BF group. (C) The IGSQ-13 score and symptom-specific score for the Lactose based and NAN group vs. the BF group.

\section{Discussion}

The present study demonstrates that $100 \%$ lactose-based formula can be well tolerated by infants aged 6-8 weeks. Formula-fed and breast-fed infants showed a statistically nonsignificant difference between the cumulative IGSQ-13 score and symptoms (stooling, spitting/vomiting, crying, fussiness, and flatulence) that are suggestive of GI distress. In this study, digestive tolerance was evaluated using the IGSQ-13 score, which is a parent report of infant GI functioning. The validity and reliability of the IGSQ score have been established through clinical research, and it was observed that the reporting is sensitive to relatively minor differences and also to the digestive tolerance symptoms between breast-fed or formula-fed infants ${ }^{[12]}$.

\section{Status of GI disorders in India}

A review published by Poddar et al. indicated that the prevalence of regurgitation in Indian infants is $55 \%$ at $1-6$ months of age, which drops to $15 \%$ by $7-12$ months, and further reduces to $10 \%$ by $12-24$ months of age. However, there are no diagnostic and management guidelines for managing infantile GI disorders in India ${ }^{[6]}$. Recently published National Health Family Survey (NFHS)-4 indicated that only $54.9 \%$ children under the age of 6 months were exclusively breastfed, whereas only $14.3 \%$ of non-breastfed children aged 6-23 months received an ageappropriate diet ${ }^{[13]}$. National Family Health Survey-4 and several other working papers on the feeding practices in India indicate that the current feeding practices in India are not nutrition-centric and age-appropriate ${ }^{[13-16]}$. In India, there is a strong need to increase the awareness about the appropriate feeding practices, guidelines, and management strategies to address GI disorders in infants.

\section{Nutrition intervention to address GI distress}

The global prevalence of GI distress due to various causes such as colic, regurgitation, and functional constipation at very young ages is relatively high ${ }^{[1,3]}$. Regrettably, the current understanding of GI diseases is hampered because of varied etiology and symptoms and, hence, no general consensus is available ${ }^{[10]}$. As conventional strategies mainly provide symptomatic relief, emerging evidence suggests that nutritional management could be considered as a promising alternative in prevention and management of GI distress ${ }^{\text {[9- }}$ 11]. Whenever breast milk is not available, ensuring nutritional intervention, which is comparable to breast milk, is necessary for optimal digestive health. It is recommended that optimal lactose content; whey/casein ratio; calcium/phosphate ratio; osmolality; and pro- and prebiotics should be considered to alleviate infantile GI distress ${ }^{[17]}$.

In this regard, IF is considered as an alternative/adjuvant source of nutrition to breast milk in infants and toddlers ${ }^{[18]}$. Some studies have observed that formula-fed infants tend to have harder stools compared with BF infants. A primary cause of harder stools can be attributed to the inadequate absorption of palmitic acid. The palmitic acid from palm ole in is at different place in IFs compared to breast milk triglyceride and is inadequately absorbed ${ }^{[19]}$ Unabsorbed palmitic acid forms calcium soaps that are insoluble at body temperature and are excreted in feces ${ }^{[19]}$. Formation of calcium soaps in the gut could be associated with the considerable differences of bowel habit and stool consistency between breastfed and formula-fed infants ${ }^{[19]}$. A recently published multicenter clinical trial observed an 
improved stool consistency and bone mineral content in infants who received formulae enriched with palmitate at the $s n-2$ position ${ }^{[20]}$. We also noticed a similar observation where hard stooling frequency and difficulty when passing a bowel movement were similar in both formula-fed and breastfed study groups. Lactose has an ability to retain water into the intestinal lumen, which results in providing osmotic laxative effect. This also could be one of the reasons for statistically non-significant difference observed between the study groups. Our choice of $100 \%$ lactose-based formula was further supported by previously published studies, where the introduction of $100 \%$ lactose-based formula resulted in considerable reduction in the constipation-related symptoms ${ }^{[21]}$.

Another nutritional intervention which is widely followed but not recommended by the guidelines is introduction of cow's milk. A joint recommendation by the American and European guideline advises the elimination of cow's milk protein, thickened feeds, reduction of ingested volume and so on as some of the non-pharmacological interventions for managing pediatric gastroesophageal diseases ${ }^{[9]}$. It is observed that, in India, breastfeeding-weaning practices predominantly include animal/cow's milk or dilution of cow's milk ${ }^{[14]}$. From nutritional perspective, cow's milk is considered as a poor source of all essential nutrients. Calcium absorption of cow's milk is $20 \%$ less than that of human milk and the lactose content is also considerably lower $(\sim 3.7-5 \mathrm{~g} / 100 \mathrm{~g})$ than that in human milk $(\sim 6.9-7.5$ $\mathrm{g} / 100 \mathrm{~g}$ ); hence, cow's milk is a comparatively suboptimal energy source ${ }^{[22,23]}$. Whey/casein ratio in cow's milk is $20: 80$, which is very low compared with the optimal ratio of 60:40 in breast milk ${ }^{[17]}$. In addition, introduction of cow's milk in infant's feed can evoke an immunological response, resulting in cow's milk protein allergy and cow's milk propensity to lead to iron deficiency, which increases the risk of dehydration ${ }^{[24]}$.

Furthermore, we did not find any statistical difference between the two groups (formula-fed vs. breastfed) with respect to other digestive symptoms, including spitting/vomiting, crying, fussiness, and flatulence, that are suggestive of GI distress. These observations are indicative that the $100 \%$ lactose-based formula might have properties closer to breast milk; hence, statistically non-significant differences were noted as the cumulative IGSQ score and occurrence of symptoms. In short, optimal nutrition that can provide gut comfort has been elicited by this study. However, future studies are needed to determine the longterm benefits of introducing 100\% lactose-based formula and its beneficial effects in providing gut comfort to larger groups of infants experiencing GI distress.

\section{Conclusion}

In conclusion, this study demonstrates that feeding infants with $100 \%$ lactose-based formula, in comparison with breastfed children, did not show any difference in various symptoms (stooling, spitting/vomiting, crying, fussiness, and flatulence) suggestive of GI distress. The GI index score was found to be similar between both study groups. Hence, lactose based-based formula can be considered as promising alternatives to the current management of infants with GI distress. However, IF should always be considered as a substitute and not a replacement to breast milk.

\section{Acknowledgments}

We would like to acknowledge the Bio Quest solutions for their assistance in drafting and editing this manuscript.

\section{References}

1. Vandenplas Y, Abkari A, Bellaiche M. Prevalence and Health Outcomes of Functional Gastrointestinal Symptoms in Infants from Birth to 12 Months of Age. JPGN. 2015; 61(5):531-7.

2. Indrio F, Mauro Di A, Riezzo G, Civardi E, Intini C, Corvaglia L et al. Prophylactic use of a probiotic in the prevention of colic, regurgitation, and functional constipation: a randomized clinical trial. JAMA Pediatr. 2014; 168(3):228-33.

3. Johnson JD, Cocker K, Chang E. Infantile colic: Recognition and treatment. Am Fam Physician. 2015; 92(7):577-82.

4. Czinn SJ, Blanchard S. Gastroesophageal Reflux Disease in Neonates and Infants. Pediatr Drugs. 2013; 15(1):19-27.

5. Koda YKL, Ozaki MJ, Murasca K, Vidolin E. Clinical features and prevalence of gastroesophageal reflux disease in infants attending a pediatric gastroenterology reference service. Arq Gastroenterol. 2010; 46(1):6671.

6. Poddar U. Diagnosis and management of gastroesophageal reflux disease (GERD): An Indian perspective. Indian Pediatr. 2013; 50:119-26.

7. Koppen IJN, Nurko S, Saps M, Di Lorenzo C, Benninga MA. The pediatric Rome IV criteria: what's new? Expert Rev Gastroenterol Hepatol. 2017; 11(3):193-201.

8. Benninga MA, Nurko S, Faure C, Hyman PE, James Roberts IS, Schechter NL Childhood functional gastrointestinal disorders: Neonate/toddler. Gastroenterology. 2016; 150(6):1443-1455e2.

9. Rosen R, Vandenplas Y, Singendonk M. Pediatric Gastroesophageal Reflux Clinical Practice Guidelines: Joint Recommendations of the North American Society for Pediatric Gastroenterology, Hepatology, and Nutrition and the European Society for Pediatric Gastroenterology, Hepatology, and Nutritio. JPGN. 2018; 66(3):516-54.

10. Savino F, Tarasco V. New treatments for infant colic. Current Opinion in Pediatrics. 2010; 22:791-7.

11. Whitfield KL, Schulman RJ. Treatment options for functional gastrointestinal disorders: From empiric to complementary approaches. Pediatr Ann. 2009; 38(5):288-94.

12. Riley AW, Trabulsi J, Yao M, Bevans KB, Derusso PA. Validation of a parent report questionnaire. Clin Pediatr (Phila). 2015; 54(12):1167-74.

13. Ministry of Health and Family Welfare Government of India. National Family Health Survey (NFHS)-4, India [Internet]. Available from: http://rchiips.org/NFHS/pdf/NFHS4/India.pdf Accessed on 30 May 2018

14. Aggarwal A, Verma S, Faridi M, Dayachand. Complementary feeding- Reasons for inappropriateness in timing, quantity and consistency. Indian $\mathrm{J}$ Pediatr. 2008; 75(1):49-53.

15. Srivastava G, Bhatnagara S, Khan K. Complementary feeding practices of children (6 months- 23 months) in 
and around Lucknow, India. Int. J Contemp Pediatr. 2018; 5(1):114-8.

16. Rao S, Swathi P, Unnikrishnan B, Hegde A. Study of complementary feeding practices among mothers of children aged six months to two years - A study from coastal south India. Australas Med J. 2011; 4(5):252-7.

17. Kesavelu D, Sethi G, Bangale N, Anwar F, Rao S. Common gastrointestinal distress among infants: Role of optimal nutritional interventions. Clin Epidemiol Glob Heal. 2018; 6(1):5-9.

18. EFSA Panel on Dietetic Products Nutrition. Scientific Opinion on nutrient requirements and dietary intakes of infants and young children in the European Union. EFSA J. 2013; 11(10):1-103.

19. Lloyd B, Halter RJ, Kuchan MJ, Baggs GE, Ryan AS, Masor ML. Formula Tolerance in Postbreastfed and Exclusively Formula-fed Infants. Pediatrics. 1999; 103(1):e7.

20. Beghin L, Marchandise X, Lien E, Bricout M, Bernet JP, Lienhardt JF et al. Growth, stool consistency and bone mineral content in healthy term infants fed sn-2palmitate-enriched starter infant formula: A randomized, double-blind, multicentre clinical trial. Clin Nutr, 2018.

21. Infante DD, Segarra OO, Redecillas SS, Alvarez MM, Miserachs MM. Modification of stool's water content in constipated infants: Management with an adapted infant formula. Nutr J. 2011; 10(1):55.

22. Chapter 3: Lactose content of milk and milk products. [Internet]. 1988 [cited 15 June 2016]; 48(4):1099-1104. Am J Clin Nutr. 1988; 48(4):1099-104.

23. Agostoni C, Decsi T, Fewtrell M, Goulet O, Kolacek S, Koletzko B et al. ESPGHAN Committee on Nutrition: Complementary feeding: A commentary by the ESPGHAN Committee on Nutrition. Journal of Pediatric Gastroenterology and Nutrition. 2008; 46:99110.

24. Ziegler E. Adverse effects of cow's milk in infants. In: Nestle Nutrition Workshop Series: Pediatric Program, 2007, 185-96. 